\title{
Planar terahertz metamaterial using triangular spiral ring resonator
}

\begin{abstract}
We report a new shape of subwavelength triangular spiral ring resonator which is designed to operate in terahertz regime. The metamaterial split ring resonator unit cell is constructed with the help of a single loop of conducting wire strip printed over a very thin high permittivity silicon dielectric substrate. The effective permittivity, permeability and simulated scattering parameters show that our proposed metamaterial can be used in number of applications in terahertz frequency band where broadband operation is required. Unloaded $\mathrm{Q}$ factor is calculated for new terahertz metamaterial resonator. The reported structure is novel in terms of its broadband operation in $\mathrm{THz}$ frequencies.
\end{abstract}

Keyword: Metamaterial; Permittivity; Permeability terahertz; Broadband; Unloaded Q 